\title{
Interplanetary shock transmitted into the Earth's magnetosheath: Cluster and Double Star observations
}

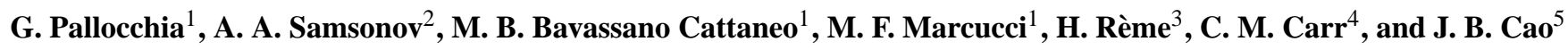 \\ ${ }^{1}$ Istituto di Fisica dello Spazio Interplanetario - Istituto Nazionale di Astrofisica, Rome, Italy \\ ${ }^{2}$ St. Petersburg State University, St. Petersburg, Russia \\ ${ }^{3}$ Centre d'Etude Spatiale Des Rayonnements, Toulouse, France \\ ${ }^{4}$ The Blackett Laboratory, Imperial College, London, UK \\ ${ }^{5}$ Center for Space Science and Applied Research, Beijing, China
}

Received: 3 September 2009 - Revised: 27 April 2010 - Accepted: 17 May 2010 - Published: 20 May 2010

\begin{abstract}
On day 7 May 2005, the plasma instruments on board Double Star TC1 and Cluster SC3 spacecraft register inside the magnetosheath, at 19:15:12 and 19:16:20 UT, respectively, a strong pressure pulse due to the impact of an interplanetary shock wave (IS) on the terrestrial bow shock. The analysis of this event provides clear and quantitative evidences confirming and strengthening some results given by past simulations and observational studies. In fact, here we show that the transmitted shock is slowed down with respect to the incident IS (in the Earth's reference frame) and that, besides the transmitted shock, the IS - bow shock interaction generates a second discontinuity. Moreover, supported also by a special set three-dimensional magnetohydrodynamic simulation, we discuss, as further effects of the interaction of the IS with the magnetosphere, other two interesting aspects of the present event, that is: the TC1 double crossing of the bow shock (observed few minutes after the impact of the IS) and the presence, only in the SC3 data, of a third discontinuity produced inside the magnetosheath.
\end{abstract}

Keywords. Magnetospheric physics (Magnetotail; Solar wind-magnetosphere interactions) - Space plasma physics (Shock waves)

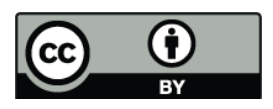

Correspondence to: G. Pallocchia (giuseppe.pallocchia@ifsi-roma.inaf.it)

\section{Introduction}

Interplanetary shocks (IS) are compressional magnetohydrodynamic (MHD) discontinuities propagating through the solar wind. The IS are known as one of the sources of geomagnetic disturbances (Tsurutani and Gonzalez, 1997). In fact, their impulsive action causes global changes in the magnetosphere determining several perturbative phenomena like, for example, sudden impulses (SI) or sudden storm commencements (SSC) (see, e.g. Huttunen et al., 2005, and references therein).

The first phase of the interaction, between an IS and the terrestrial magnetosphere, consists of the IS collision on the bow shock. Theoretically, this process is highly nonlinear. Actually, on the contact line of the two shock fronts, it is generally impossible to satisfy the Rankine-Hugoniot jump conditions with only one MHD discontinuity. Therefore, this discontinuity splits up immediately in an ensemble of other discontinuities or self-similar waves (Akhiezer et al., 1975). The most general solution is given by a combination of seven discontinuities and rarefaction waves: three waves travelling in a direction (in this sequence: fast, Alfvén and slow) and three waves travelling in the opposite direction (in the same sequence) separated by a contact discontinuity, at rest relative to the medium (Jeffrey and Taniuti, 1964). The fast and slow waves can be either shocks or rarefaction waves. Of course, some of the waves enumerated here may not be present in the solutions of particular problems.

In the past years several theoretical papers were devoted to the interaction of a IS with the bow shock. The pioneering one-dimensional studies, in gasdynamical approximation or with a magnetic field perpendicular to the solar

Published by Copernicus Publications on behalf of the European Geosciences Union. 
wind velocity and to the shock normals, have shown that the collision, between a fast IS and the bow shock, produces a forward-reversed couple of fast shocks separated by a contact discontinuity (Shen and Dryer, 1972; Dryer, 1973; Grib et al., 1979).

By taking into account a magnetic field direction forming a $45^{\circ}$ angle with the solar wind velocity, Grib (1982) has found that the interaction of a fast IS with the bow shock results in a couple of forward waves (i.e., the transmitted fast IS and a slow expansion wave), a couple of reverse waves (i.e., the fast modified bow shock and a slow shock) and a contact discontinuity. The Grib (1982) theoretical findings have been confirmed by later MHD simulations both in one-dimensional (Yan and Lee, 1996) and in full three-dimensional case close to the Sun-Earth line (Samsonov et al., 2006). Moreover, Samsonov et al. (2006) have shown that the speeds of the forward slow expansion wave, of the contact discontinuity and of the reverse slow shock are all very close. In the experimental data, therefore, a compound discontinuity (i.e. a discontinuity resulting from two or more superimposed waves or discontinuities) rather than the three separate waves should be probably observed. Some evidencies supporting this issue can be found in past papers (Šafránková et al., 2007; Přech et al., 2008) and, as it will be discussed, also in the present paper. However, with regard to the problem of the number of discontinuities in the ensemble resulting from the IS-bow shock collision, it should be stressed that the nature of this ensemble may change if the parameters, concerning the initial conditions of the model, are modified (Pushkar' et al., 1991).

The second phase of the process of interaction of a IS with the magnetosphere consists of the propagation of the shock front through the magnetosheath. Calculating the differences between the predicted and the observed times of arrival of 10 interplanetary shocks to the spacecraft locations in the magnetosheath, Koval et al. (2006a) have found a deceleration of the IS within the magnetosheath ranging from 0.82 to 0.97 of the shock speed in the solar wind. A deceleration, even if much higher (a shock speed in the magnetosheath ranging from 0.25 to 0.33 of the external speed), is also reported in an observational study on 20 cases by Villante et al. (2004). At present, the geometry of the shock front in the magnetosheath is still matter of discussion. Using a hydrodynamic model, Spreiter and Stahara (1994) have found that the shock front is still nearly planar. Differently, by means of observations from several spacecraft and of MHD modeling, Koval et al. $(2005,2006 \mathrm{~b})$ have argued that the transmitted IS front has a curved profile within the magnetosheath.

The collision on the magnetopause is another crucial phase in the interaction of an IS with the magnetic Earth's environment and it is, as the other two previously mentioned phases, still poorly understood. In a theoretical study based on the Rankine-Hugoniot relations, Grib et al. (1979) have shown that the impact of a fast shock on the magnetopause, considered as a tangential discontinuity, produces a fast rarefaction wave moving sunward. Grib and Martynov (1977) have proposed that this reflected rarefaction wave starts an oscillating process in which other secondary waves are generated by the reflections upon both the bow shock and the magnetopause. This mechanism produces oscillations of the magnetopause and bow shock positions and also reverse shocks in the magnetosheath due to the evolution of outward compression waves. Global MHD simulations (Samsonov et al., 2007) show that the interaction of a fast shock with the magnetopause results in a transmitted fast shock propagating earthward through the magnetosphere. This transmitted shock reflects from the inner boundary of the numerical model which may be either the plasmapause or the ionosphere. The reflected fast shock propagates sunward through the dayside magnetosphere and magnetosheath. The passage of the transmitted shock causes the bow shock and magnetopause to move inward, while the passage of the reflected fast shock causes these boundaries to move outward.

Here, we present an event in which both Cluster and Double Star spacecraft register, in quite different positions within the magnetosheath, a pressure pulse due to an IS impinging on the bow shock. Our analysis provides some interesting quantitative results regarding the motion of the transmitted IS, of the bow shock and two secondary discontinuities generated by the IS-magnetosphere interactions. Moreover, we discuss our results also with the support of a specially set simulation based on the local model by Samsonov et al. (2006)

\section{Double Star and Cluster observations}

The event under study occurs on 7 May 2005 between 19:13 and 19:23 UT. The data used are from Hot Ion Analyser (HIA) and Flux Gate Magnetometer (FGM) onboard Cluster SC3 and Double Star TC1 spacecraft. HIA instrument selects the incoming ions according to the ion energy per charge ratio by electrostatic deflection in an analyser having a "top hat" geometry (Carlson et al., 1982). The particle imaging is based on microchannel plate (MCP) electron multipliers and position encoding discrete anodes. HIA is able to provide, in a spacecraft spin period of $\sim 4 \mathrm{~s}$, a full threedimensional ion distribution function in the energy range of 5 to $32000 \mathrm{eV}$, with no mass separation. FGM flight instrumentation consists of two, tri-axial fluxgate magnetometer and an onboard data-processing unit on each spacecraft, and is capable of high sample rates (up to 67 vectors $\mathrm{s}^{-1}$ ) at high resolution (up to $8 \mathrm{pT}$ ). Cluster (Cluster Ion Spectrometry (CIS)/HIA and FGM) and Double Star (HIA and FGM) experiments are widely described in Rème et al. (2001) and Balogh et al. (2001), Rème et al. (2005) and Carr et al. (2005), respectively. For the present study, HIA onboard moments of the ion distribution function and spin averaged magnetic field are used and, therefore, the time resolution of the data set is $\sim 4 \mathrm{~s}$. Unfortunately, in this event, other HIA data from Cluster are not available due to some data gaps (SC1) 
and to a not suitable operation mode (SC4) in the considered time interval whereas no HIA data are available on SC2 at all.

On day 7 May 2005, the Hot Ion Analysers (HIA) plasma instruments, onboard Double Star TC1 and Cluster SC3 spacecraft, start to measure, at 19:15:12 and 19:16:20 UT, respectively, a pressure pulse (Fig. 1). At those times, both satellites are on dawn flank and in the northern magnetosheath, with TC1 closer both to the bow shock and to the Sun-Earth line than SC3 (Fig. 2).

This compression is due to the impact of an interplanetary discontinuity on the terrestrial bow shock. Indeed, the ACE monitor observes, at 18:18:32 UT and near the lagrangian point $\mathrm{L} 1$, an abrupt increase in the protons density $n$, temperature $T$ and bulk flow speed $V$. The magnetic field $\boldsymbol{B}$ rotates of a very small angle whereas its magnitude $B$ jumps (Fig. 3 and Table 1). Given that, across a MHD discontinuity whose normal is $\boldsymbol{n}, \boldsymbol{B} \cdot \boldsymbol{n}$ is conserved (e.g., Landau and Lifshitz, 1960), it is easily deduced that, in the present case, $\boldsymbol{B}$ is nearly perpendicular to the normal discontinuity. The above features, together with the observation that the jump $n_{2} / n_{1}$ is very close to $B_{2} / B_{1}$ (Table 1 ) (the subscripts 1 and 2 will refer, from now on, to the upstream and downstream average parameters, respectively), permit to identify the interplanetary discontinuity as a fast quasi-perpendicular shock (e.g., Hudson, 1970). The shock normal at the ACE location is $\boldsymbol{n}_{(\mathrm{IS})}(\mathrm{ACE})=(0.982,0.172,-0.08)$ and the IS speed (in the spacecraft reference frame) is $U_{\mathrm{n}_{(\mathrm{IS})}}(\mathrm{ACE})=461 \mathrm{~km} / \mathrm{s}$ earthward. The upstream fast magnetosonic Mach number has a value of $M_{\mathrm{f} 1}=1.25$ indicating that the IS is a quite weak shock. The estimated propagation time, from ACE to the $\mathrm{TC} 1$ position, results to be $55.13 \mathrm{~min}$, in good agreement with the observed lag of $56.7 \mathrm{~min}$. Therefore, there exists a strict relation between the arrival of the IS and the observed compression of the magnetosheath.

The pressure pulse shows clearly a compound structure inside the magnetosheath. In fact, TC1 resolves the whole perturbation as two distinct discontinuities separated by $\sim 30 \mathrm{~s}$ in time (Fig. 1a). The first discontinuity, at 19:15:12 UT, is characterized by an increase of $n, T$ and also of $V$ (Fig. 1a). Moreover, the magnetic field rotates of a small angle of $5.5^{\circ}$ whereas its magnitude $B$ slightly increases (Table 1). This discontinuity represents again a quasi-perpendicular shock wave which is actually the transmitted IS. The TC1 data analysis shows that the transmitted shock is earthward directed with a speed of $U_{\mathrm{n}_{(\mathrm{IS})}}(\mathrm{TC} 1)=328 \mathrm{~km} / \mathrm{s}$ along its normal $\boldsymbol{n}_{\text {(IS) }}(\mathrm{TC} 1)=(0.992,0.125,-0.025)$ whereas the upstream fast magnetosonic Mach number is $M_{\mathrm{f} 1}=1.01$.

The second discontinuity, observed by TC1 at 19:15:40, is charaterized by a decrease of $T$ and a weak increase of $n$. On the contrary, $\boldsymbol{B}$ is practically constant through it (Fig. 1a and Table 1). A decrease of the y-component of the plasma bulk flow velocity $\left(V_{2_{Y(\mathrm{GSE})}} / V_{1_{Y(\mathrm{GSE})}}=0.6\right)$ is also observed $\sim 8-12$ s before the $T$ decrease. However this finding must a) TC1

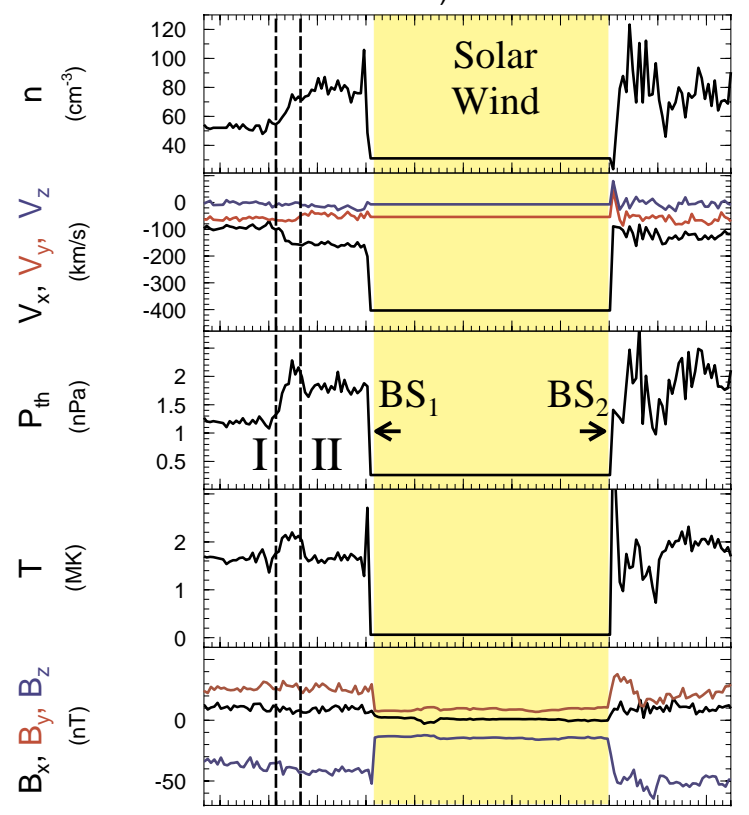

b) SC3

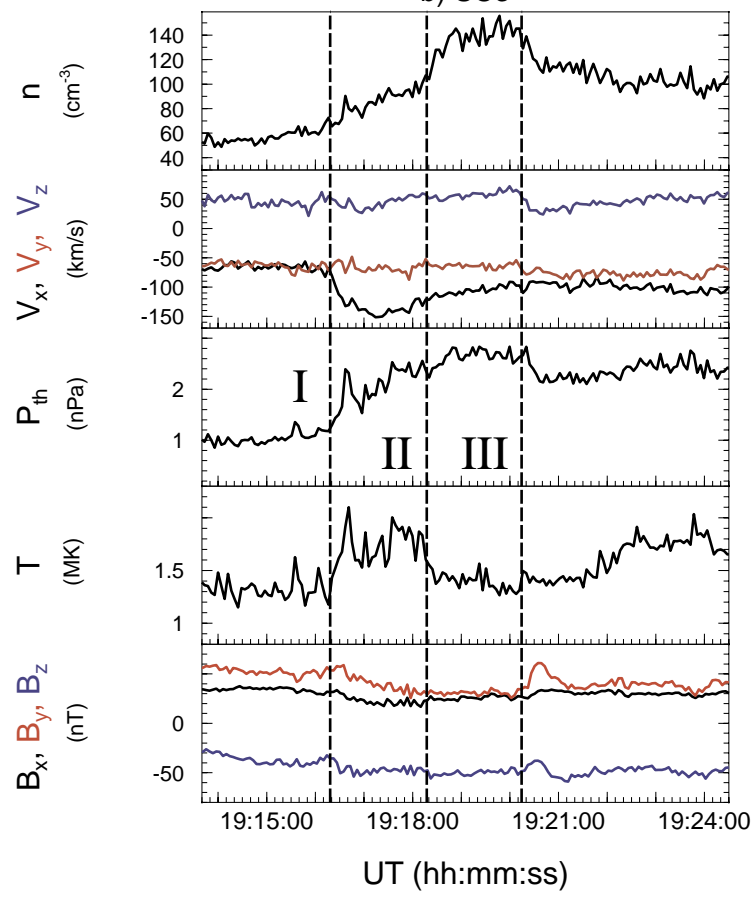

Fig. 1. Ions parameters and magnetic field for the present event as observed by: (a) TC1, (b) SC3. $\boldsymbol{V}$ and $\boldsymbol{B}$ are given in GSE coordinates. The vertical lines I, II and III indicate the discontinuities discussed in the text. In-between the outbound $\mathrm{BS}_{1}$ and the inbound $\mathrm{BS}_{2}$ bow shock crossings, TC1 is in the solar wind. Due to a saturation of the HIA instrument in the solar wind, the plasma parameters plotted in the yellow area in a) are constant values obtained by time averaging ACE/SWEPAM data over a suitable interval in the ACE post-IS region. 


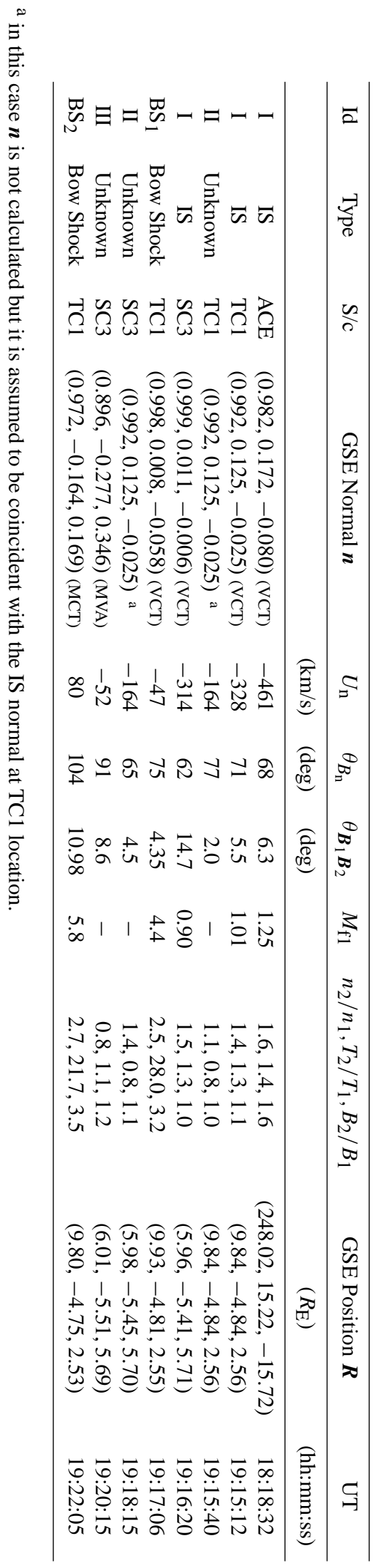

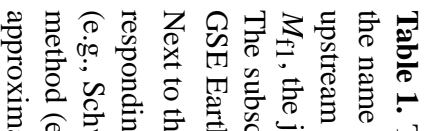

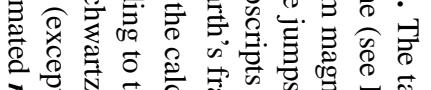

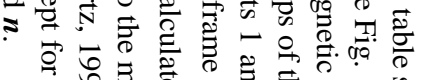

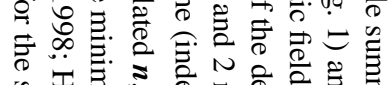

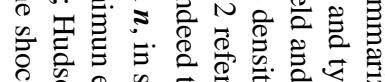

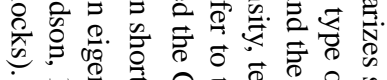

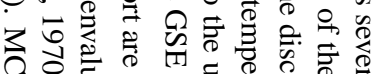

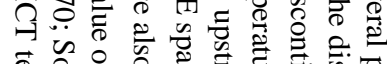

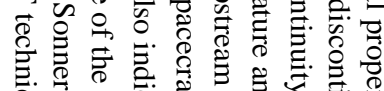

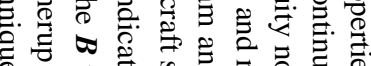

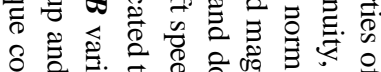

ठํ.

टि 0 है

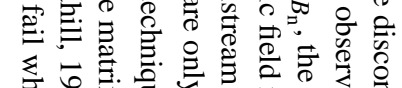

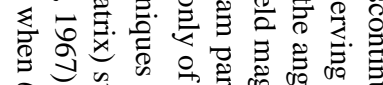

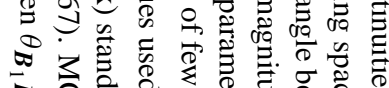

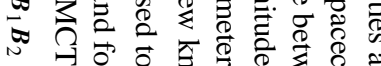

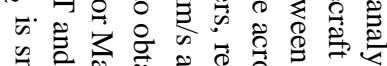

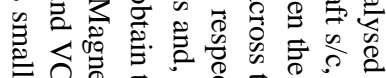

ㄱㄱㅇㅠ.

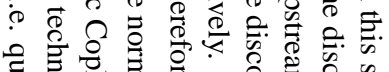

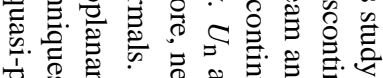

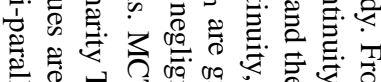

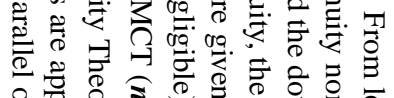

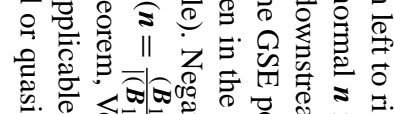

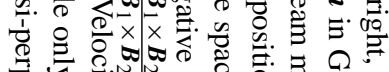

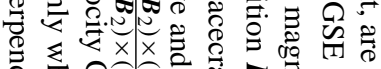

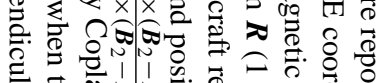

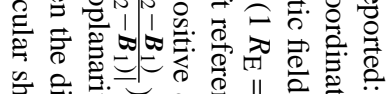

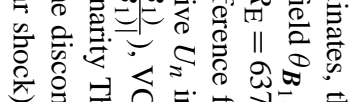

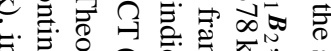

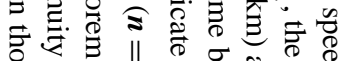

到

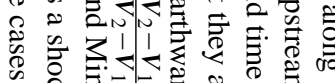

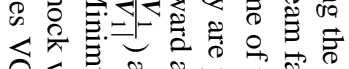

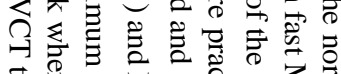

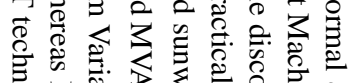

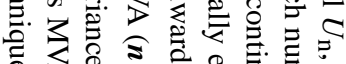

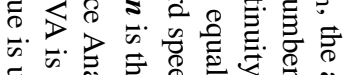

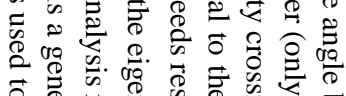

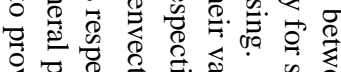

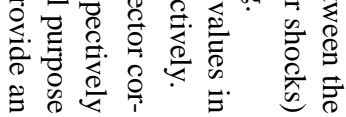



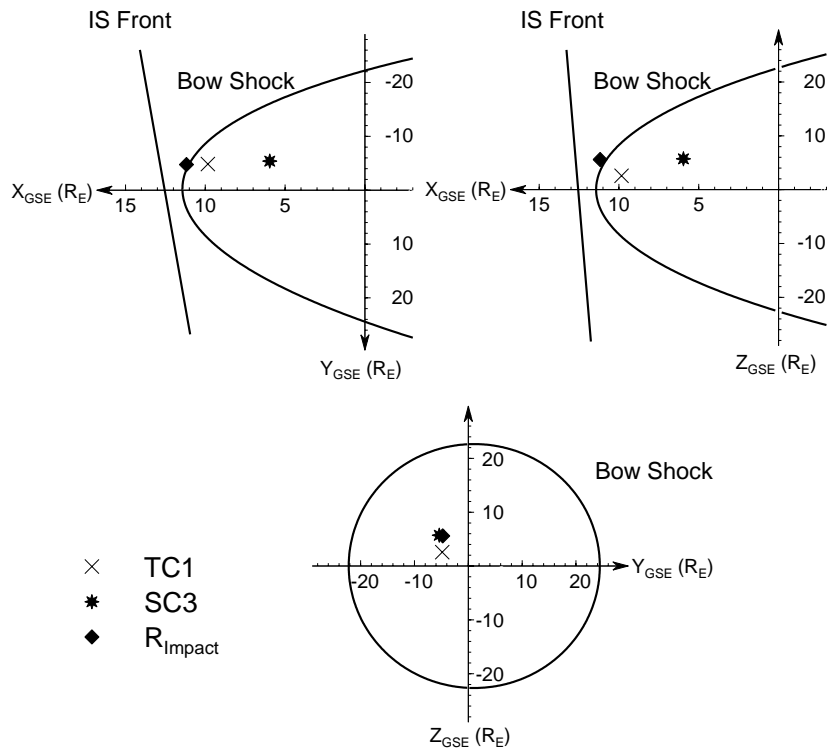

Fig. 2. Spacecraft, IS front and the Earth's bow shock positions just before the IS impact. The reconstructed impact point $R_{\text {impact }}$ is also drawn. The model bow shock location (by Formisano, 1979) has been calculated using the pre-IS solar wind dynamical pressure $\varrho V^{2}=4.7 \mathrm{nPa}$ and the alfvénic Mach number $M_{\mathrm{A}}=7.4$.

be considered with caution because the parameters jumps are quite weak and the fluctuations not negligibile.

The SC3 spacecraft is positioned more deeply into the magnetosheath, roughly 4 Earth's radii along the Sun-Earth line, with respect to the TC1 spacecraft (Fig. 2). When seen by $\mathrm{SC} 3$, the structure of the magnetosheath perturbation consists of three discontinuities (Fig. 1b).

The first of these, observed at 19:16:20 UT, is still the quasi-perpendicular fast transmitted shock whose jumps of the $n, T$ and $B$ are all quite close to the ones measured at the TC1 location (Table 1). In this case, the measured speed of IS has a value of $U_{\mathrm{n}_{(\mathrm{IS})}}(\mathrm{SC} 3)=314 \mathrm{~km} / \mathrm{s}$ whereas the shock normal is practically coincident with the $X_{\mathrm{GSE}}$ axis (Table 1). Using the time delay of $\sim 68 \mathrm{~s}$, between the shock arrivals at TC1 and SC3 locations, and the TC1-SC3 separation of $\sim 4.0 R_{\mathrm{E}}$ along the shock normal $\boldsymbol{n}_{\text {(IS) }}(\mathrm{TC} 1)$, we obtain an average shock speed of $\left\langle U_{\mathrm{n}_{(\mathrm{IS})}}\right\rangle=375 \mathrm{~km} / \mathrm{s}$ earthward which is close to both values of $328 \mathrm{~km} / \mathrm{s}$ and $314 \mathrm{~km} / \mathrm{s}$, i.e. the Rankine-Hugoniot istantaneous values of the IS speed at TC1 and SC3 positions respectively. With regard to the Cluster observations of the IS, it is necessary to explain the reason why the multi-spacecraft timing technique (e.g., Schwartz, 1998) has not been used to measure the normal and speed of the shock. Actually, the magnetic field data are available also from SC1, SC2 and SC4 but the time delays (of the IS arrivals between the four spacecraft) are very poorly measured by means of the FGM data inspection. The reason of this drawback is that those delays are short, at most

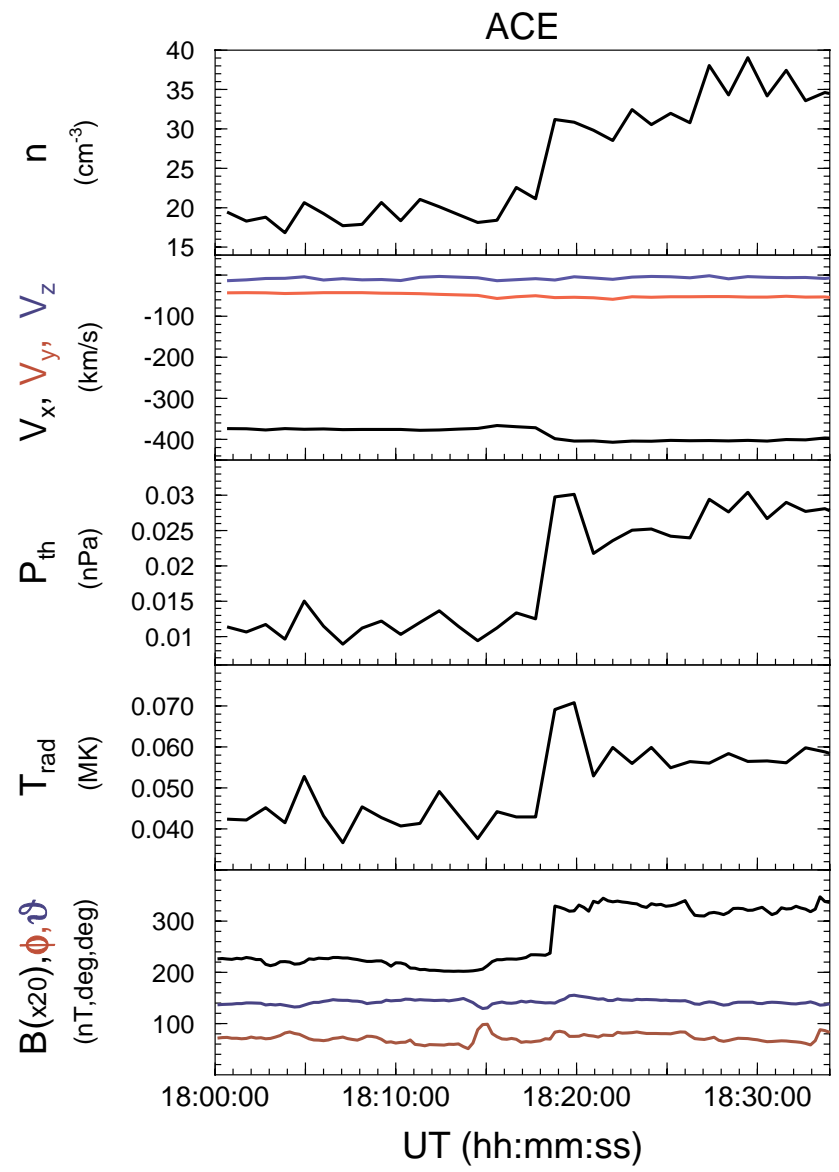

Fig. 3. The perpendicular interplanetary shock, causing the magnetosheath perturbation of Fig. 1, as seen by ACE/SWEPAM (plasma analyzer) and ACE/MAG (magnetometer) instruments. The time resolutions of plasma and magnetic field L2 verified data are $64 \mathrm{~s}$ and $16 \mathrm{~s}$, respectively. The magnetic field is reported in GSE polar coordinates. $T_{\mathrm{rad}}$ is the proton radial temperature.

of few seconds, and the IS magnetic signatures (i.e. $\boldsymbol{B}$ rotation and $B$ jump) weak and blurred by other fluctuations with considerable amplitudes.

The second discontinuity is observed around 2 min after the transmitted shock and shows an increase of $n$ and a decrease of $T$ (Fig. 1b). Across it, moreover, $\boldsymbol{B}$ has a rotation of few degrees associated with a small jump of $B$ (Table 1). On the contrary, the flow speed field has not a discontinuous variation but a quite regular increase $(\sim 40 \mathrm{~km} / \mathrm{s})$ of $V_{\mathrm{X}(\mathrm{GSE})}$ between 19:17:40 and 19:19:30 UT (Fig. 1b). Under the hypothesis that the discontinuity is moving in the same direction as the transmitted shock $\boldsymbol{n}_{\text {(IS) }}$ (TC1), we can affirm that it is not a propagating structure. In fact, taking into account its travelling time between TC1 and SC3 $(\sim 155 \mathrm{~s})$ and the spacecraft distance along $\boldsymbol{n}_{(\mathrm{IS})}(\mathrm{TC} 1)\left(\sim 3.98 R_{\mathrm{E}}\right)$, we can estimate its average speed being $\left\langle U_{\mathrm{n}(\mathrm{II})}>=164 \mathrm{~km} / \mathrm{s}\right.$ earthward, practically the same value as the flow speed along 
$\boldsymbol{n}_{\text {(IS) }}$ (TC1). A further and extremely important annotation is that this discontinuity is not from the interplanetary space. In fact, an inspection of the solar wind data does not reveal any signature which can be associated with the present magnetosheath structure (actually, in the ACE data a drop of $T$ is visible around $2 \mathrm{~min}$ after the IS passage (Fig. 3). However, it is possible to rule out easily the possibility that the ACE $T$ drop is related to the magnetosheath discontinuities II. The argument is the following: if the ACE $T$ drop were an advected structure, then it should arrive at the Earth around $\sim 9$ min after the IS, that is a time delay much longer than the observed 2 min at SC3 location. Viceversa, if ACE $T$ drop were rigidly linked to the IS structure and, therefore, if it travelled at the same IS speed, well then its time delay from IS in magnetosheath would be consistent with the SC3 observations (delay time of $\sim 2 \mathrm{~min}$ ) but absolutely in disagreement with TC1 observations where the time delay is only of $\sim 30 \mathrm{~s}$. Moreover, it is noted that the ACE $T$ drop coincides with a density decrease whereas in the magnetosheath the $T$ decrease is associated with a density increase both at TC1 and $\mathrm{SC} 3$ positions.)

Assuming that the second discontinuity is generated when the IS hits the bow shock, it is possible to provide an approximation of both the position $\boldsymbol{R}_{\text {impact }}$ where the observed discontinuity fronts have been generated and the time $T_{\text {impact }}$ of this generation. In fact, given the speeds $<U_{\mathrm{n}_{(\mathrm{S})}}>$ and $<U_{\mathrm{n}(\mathrm{II})}>$ of the transmitted IS and of the second discontinuity respectively, given their arrivals time and also the SC3 position, a simple calculation provides the value of $\boldsymbol{R}_{\text {impact }}=$ $(11.18,-4.75,5.58) R_{\mathrm{E}}$ and places $T_{\text {impact }}$ at 19:14:51 UT. These results will be useful in the paper later on.

The third discontinuity is seen by SC3 at 19:20:15 UT and, as in the case of the second discontinuity, its interplanetary origin must be excluded. Across it, $n$ decreases whereas both $T$ and $B$ slightly increase (Fig. $1 \mathrm{~b}$ and Table 1 ). The rotations of $\boldsymbol{B}$ and $\boldsymbol{V}$ field are both less than $10^{\circ}$. The minimum variance direction of the magnetic field (Sonnerup and Cahill, 1967) $\mathbf{n}_{\mathrm{III}}=(0.896,-0.277,0.346)$, calculated in the time interval from 19:19:20 UT to 19:21:30 UT comprising this discontinuity, is very well defined being quite high the ratio between the intermediate $\lambda_{2}$ and the minimun eigenvalues $\lambda_{3}$ of the variance matrix $\left(\lambda_{2} / \lambda_{3}=31\right)$. Along $\boldsymbol{n}_{\mathrm{III}}$ the flow speed has an average component of $-52 \mathrm{~km} / \mathrm{s}$ (Fig. 4). A simple analysis of the arrival times of the discontinuity at the Cluster spacecraft positions, made with CLUSTER/FGM magnetic field data, permits to understand in which direction it is travelling. As shown in the upper panel of Fig. 5a, the four time profiles of $B_{\mathrm{y}}$ of the discontinuity are well separated in time. By means of time shifts, derived under the assumption that the discontinuity is moving earthward along $\boldsymbol{n}_{\text {III }}$ with a speed of $U_{\text {n(II) }}=52 \mathrm{~km} / \mathrm{s}$, these profiles can be nicely made overlapping (Fig. 5a). Therefore this discontinuity is an advected structure moving earthward at the local plasma speed. This last finding is also confirmed by a multispacecraft timing analysis performed on the magnetic field

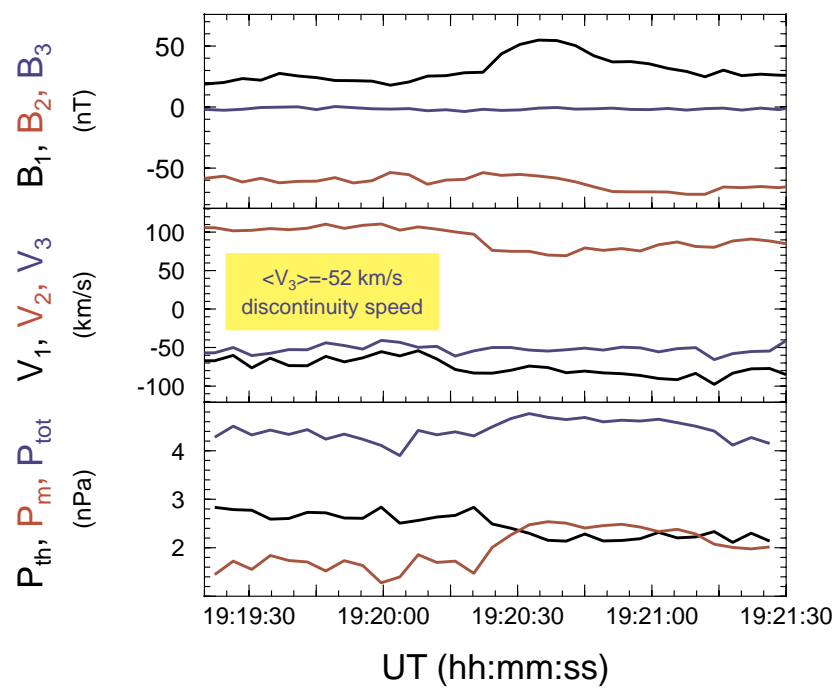

Fig. 4. The magnetic field and the bulk flow velocity for the third discontinuity observed by SC3 (see text). Both vector fields are given in the minimum variance reference frame of the magnetic field. The indices 1, 2 and 3 refer to the maximum, intermediate and minimum variance directions respectively. In the lower panel are plotted the protons thermal pressure $P_{\text {th }}$ (black line), the magnetic pressure $P_{\mathrm{m}}$ (red line) and the total pressure $P_{\mathrm{tot}}=P_{\mathrm{th}}+P_{\mathrm{m}}$ (blue line).

data. In fact, this technique provides a speed of $\sim 58 \mathrm{~km} / \mathrm{s}$ along a direction which forms an angle of only $\sim 8^{\circ}$ with $\boldsymbol{n}_{\text {III }}$. Finally, taking into account that along the $\boldsymbol{n}_{I I I}$ the magnetic field has a component nearly zero and that the total pressure is approximately balanced (Fig. 4), we note that the present structure resembles a tangential discontinuity (e.g., Landau and Lifshitz, 1960; Hudson, 1970).

Finally, we discuss another interesting aspect of the present event concerning the behaviour of the bow shock just after the impact with the IS. At 19:17:06 UT, TC1 has an outbound bow shock crossing, followed, 5 min later, by an inbound crossing (Fig. 1a). In the solar wind plasma and magnetic field behind the IS, there are no significant variations which could cause the observed double displacements of the bow shock. Therefore, the double crossing should be related to the interaction IS-magnetosphere as suggested by Šafránková et al. (2007) in the discussion of some other events quite similar to the present one. In the first crossing, we find the bow shock moving earthward with a speed of $U_{n_{\left(\mathrm{BS}_{1}\right)}}=47 \mathrm{~km} / \mathrm{s}$. Differently, in the second crossing, it turns out that the bow shock travels in the opposite way, that is sunward, with a speed of $U_{\mathrm{n}_{\left(\mathrm{BS}_{2}\right)}}=80 \mathrm{~km} / \mathrm{s}$. For sake of completeness, note that, due to the saturation of TC1 HIA instrument in the solar wind in the present event, in the above calculation of the bow shock speeds we have used suitable time averages of $n$ and $\boldsymbol{V}$ coming from ACE/SWEPAM ions instrument (Fig. 1a). The reconstucted impact time $T_{\text {impact }}$ of 
a)

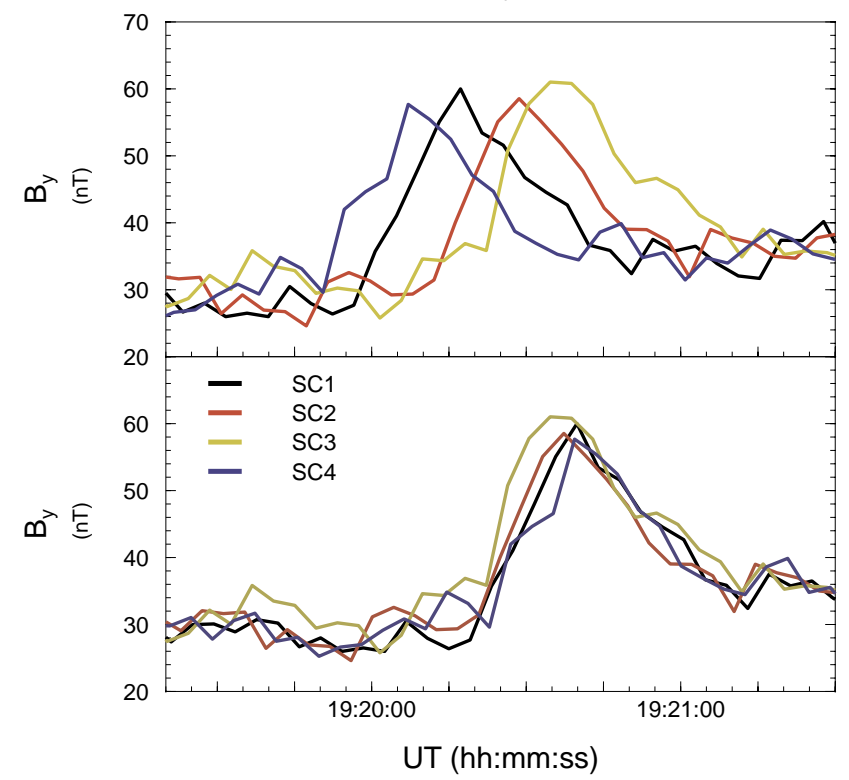

b)
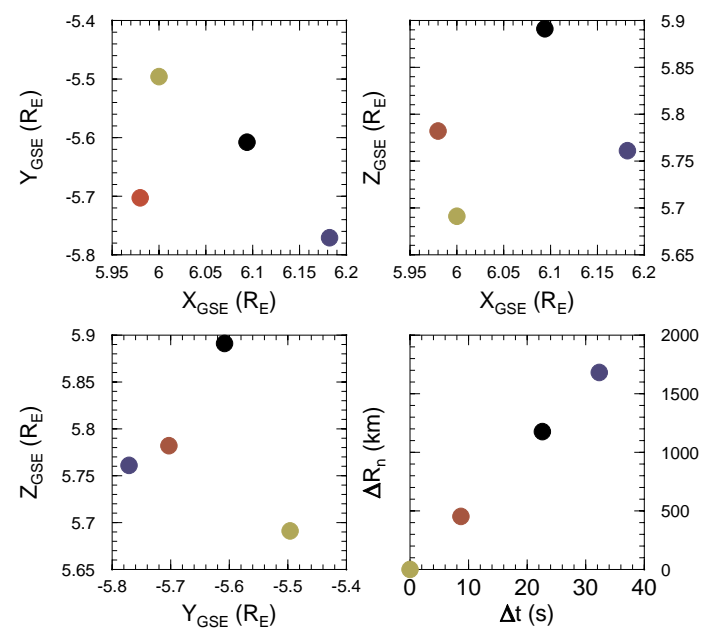

Fig. 5. (a) Upper panel: the GSE $B_{\mathrm{y}}$ component of the magnetic field of the third discontinuity as seen by the four Cluster spacecraft. Bottom panel: same as in the upper panel but with the SC1, SC2 and SC4 profiles of $B_{\mathrm{y}}$ suitably shifted ahead in time (only the $\mathrm{SC} 3$ profile is unchanged).

(b) First three panels: Cluster spacecraft GSE positions at the time of the third discontinuity passage. Last panel: the spacecraft distances from SC3, calculated along the $\boldsymbol{n}_{\text {III }}$ direction (see Table 1), plotted versus the time delays expected under the hypothesis that the third discontinuity is moving along $\boldsymbol{n}_{\mathrm{III}}$ with the local plasma speed of $U_{\mathrm{n}_{\text {(III) }}}=-52 \mathrm{~km} / \mathrm{s}$ (Table 1 and Fig. 4). The amounts of these delays equal the time shifts applied to the $B_{\mathrm{y}}$ profiles in the bottom panel of (a).

IS with the bow shock is, as previously found, 19:14:51 UT, moreover TC1 crosses the bow shock for the first time around $\sim 135$ s later. Under the hypothesis that the bow shock starts to move inward immediatly after the IS collision, taking into account also the TC1 distance of $\sim 1.17 R_{E}$ (along its normal $\left.\boldsymbol{n}_{\mathrm{BS}_{1}}\right)$ from $\boldsymbol{R}_{\text {impact }}$, we get an average speed of $\left\langle U_{\mathrm{n}_{\left(\mathrm{BS}_{1}\right)}}>=\right.$ $55 \mathrm{~km} / \mathrm{s}$ which is very close to the Rankine-Hugoniot istantaneous value of $U_{\mathrm{n}_{\left(\mathrm{BS}_{1}\right)}}=47 \mathrm{~km} / \mathrm{s}$.

\section{Numerical simulation}

We have simulated the event on 7 May 2005 using a threedimensional (3-D) MHD model developed for the Earth's magnetosheath (Samsonov et al., 2006; Samsonov, 2006). This model calculates the MHD flow around a parabolic obstacle using solar wind temporal variations observed by ACE (or another solar wind monitor) as boundary conditions at the inflow boundary. The numerical calculations are based on the TVD Lax-Friedrichs II-order scheme (e.g., Tóth, 1996) and the maximal resolution is in the subsolar region where the grid spacing equals nearly $0.2 R_{\mathrm{E}}$. The jump conditions through the bow shock are obtained self-consistently during the simulations. Since the model does not simulate the magnetospheric field, it can not take into account selfconsistently the magnetopause motion. Therefore, for the simulations in this work, the magnetopause is considered as a solid impenetrable obstacle. The reason of this assumption is that Samsonov's model, using a solid magnetopause, agrees better with magnetosheath observations of IS than the same model with moving magnetopause (e.g., Koval et al., 2006b). In this approach, the magnetopause motion can be taken into account indirectly as explained below. We record temporal variations of the MHD parameters in two points approximately corresponding to the SC3 and TC1 positions. Since the size of the magnetopause obstacle changes responding to pressure variations in front of the magnetopause, we vary the position of the artificial spacecraft in relation to the magnetopause and bow shock. In other words, it means the variations of spatial units or normalization in the simulation assume that the magnetopause shape is fixed during the considered time interval.

We start the simulation using solar wind conditions at 18:00 UT that is $18 \mathrm{~min}$ before the IS arrival at ACE. At the beginning, a stationary solution for the initial conditions has been found by the relaxation method. Then time varying boundary conditions determined by the ACE data are used. The numerical predictions (red lines) and the observed variations (black lines) of $n, V, T$ and $B$ for $\mathrm{SC} 3$ and TC1 are shown in Fig. 6 and Fig. 7, respectively. We mark three notable changes by vertical dashed lines in Fig. 6 . The first one is the IS itself when the $n, V$ and $T$ increase. $B$ increases too, but this increase is weak and is followed by a larger decrease. The second structure corresponds to another increase of $n$, but a decrease of $V$ and $T$. B slightly increases again. The third vertical line marks a decrease of $n$ and an increase of $B$ (only in the observations). The model predicts a decrease of $B$ between 19:16 and 19:17 which is really observed a 
little later in SC3 FGM data. This decrease could be caused by the inward magnetopause motion or by a fast rarefaction wave reflected from the magnetopause (Grib et al., 1979). However, the presence of a rarefaction wave does not agree with a smooth increase of $n$.

Most important features in Fig. 7 are the forward IS marked by a vertical dashed line and following double bow shock crossings when $\mathrm{TC} 1$ goes from the magnetosheath to the solar wind at 19:17 UT and then returns back into the magnetosheath $5 \mathrm{~min}$ later. Numerical simulations may help to resolve spatial-temporal uncertainty existing in spacecraft observations. Figures 8-11 show changes of the $n, V, T$ and $B$ in 24 16-s intervals in the noon-meridional plane. The panels contain only a dayside part of the numerical region where the positions of SC3 and TC1 are shown by star and cross, respectively. A decrease of parameters during an interval is shown by violet and blue colours, an increase is shown by red and yellow colours. Arrows mark the direction of motion of the bow shock (BS), interplanetary shock (IS), compound discontinuity (CD), and reflected fast shock (RFS). The IS front in the supersonic solar wind is sometimes vague because the changes are smoothed by the numerical viscosity. But it is well defined after the interaction with the bow shock when the IS goes through the dayside magnetosheath. The shock front in the magnetosheath is slightly curved as discussed by Koval et al. (2005). The interaction between the IS and the bow shock results in anti-sunward bow shock motion. The motion means a decrease of $n, T, B$ and an increase of $V$ in the points shifted from the magnetosheath to the solar wind. This helps to identify the position of the moving bow shock rather well. While the IS propagates through the inner magnetosheath, another discontinuity with an increase of $n$ and a decrease of $T$ becomes visible between the IS and the bow shock (panels 7-8 of Figs. 8 and 11). In a particular case simulated by Yan and Lee (1996) and Samsonov et al. (2006), this discontinuity was found to be a combination of a forward slow expansion wave, a contact discontinuity, and a reversed slow shock. The three discontinuities travel with similar speeds and can not be resolved in the 3-D simulations (Samsonov et al., 2006). However, another combination of discontinuities may appear for a slightly different upstream configuration (see results of Pushkar' et al., 1991; Grib and Pushkar, 2006). The contact discontinuity seems to exist in any combination, and it is generally surrounded by slow shocks and/or slow rarefaction waves. Panels $7-8$ of Fig. 10 show two thin layers in the subsolar region with decreased and increased $B$ shifted earthward and sunward with respect to the variation of $n$ and $T$ which would agree with the combination of a forward slow shock, a contact discontinuity and a reversed slow shock. The changes of $B$ through the slow shocks are relatively small and can not be identified clearly at both spacecraft positions. A stronger anti-phase variation of $n$ and $T$ caused by a contact discontinuity has been observed by SC3 (second vertical line) and predicted in the simulation (between 19:18 and 19:19 in Fig. 6). This dis-

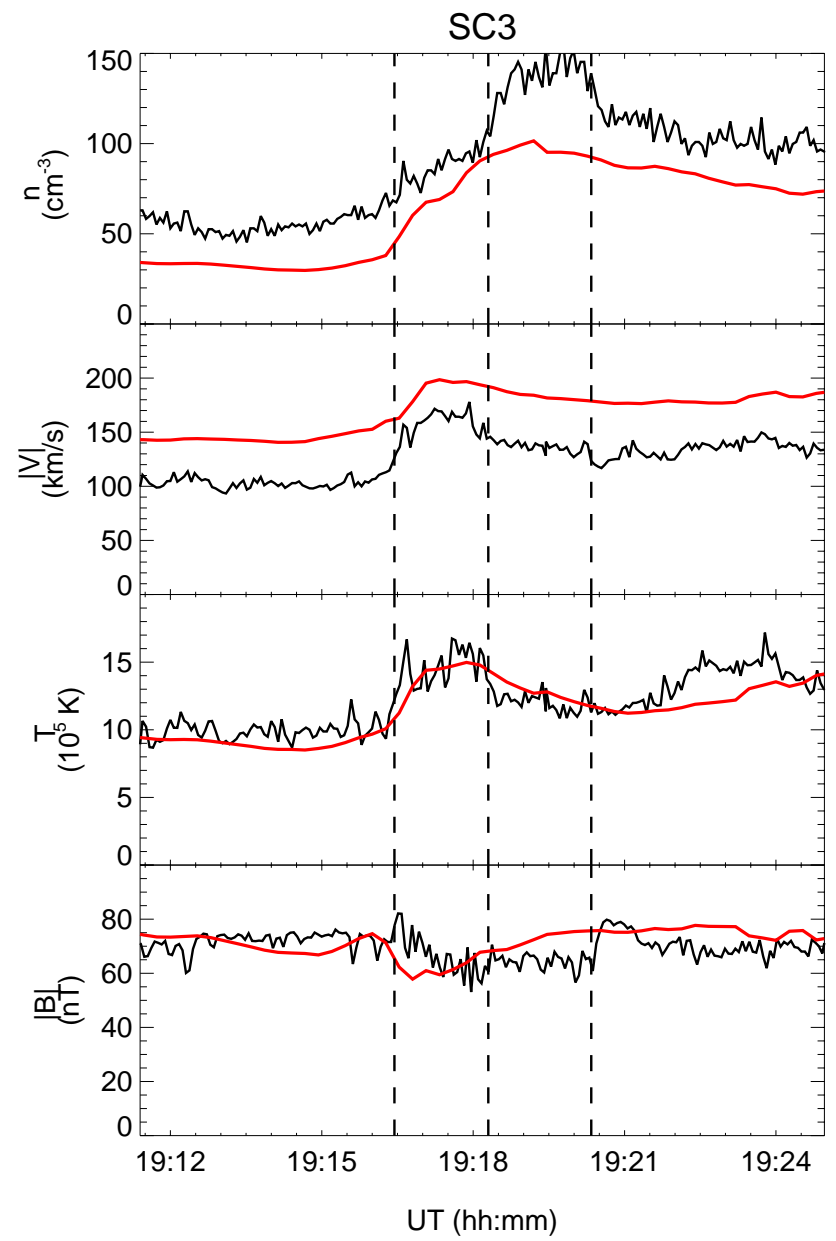

Fig. 6. Ions density, total speed, temperature and magnetic field magnitude observed by CIS/HIA and FGM instruments onboard SC3 (black lines) and predicted by the MHD code (red lines). Vertical dashed lines mark the three observed discontinuities (see details in text).

continuity is also found in the simulated TC1 profile although it is then overlapped by the outbound bow shock crossing. In fact, $T$ decreases at around 19:16 well before $n$ or $B$ do and, when $T$ begins decreasing, $n$ has a second step of increase (Fig. 7). Therefore, the jumps of some parameters are not simultaneous indicating that this discontinuity has a compound nature.

The incident fast shock reflects from the inner numerical boundary in the simulation producing a reverse fast shock (RFS) which propagates toward the bow shock. The RFS is well defined in the all figures (panels 8-13 of Figs. 8-11).

When the RFS reaches the bow shock, this begins to move sunward. The sunward bow shock motion appears first near the Sun-Earth line, while the flank bow shock still moves anti-sunward (panels 14-17). Then the sunward moving region of the bow shock extends toward the flanks. Similar 


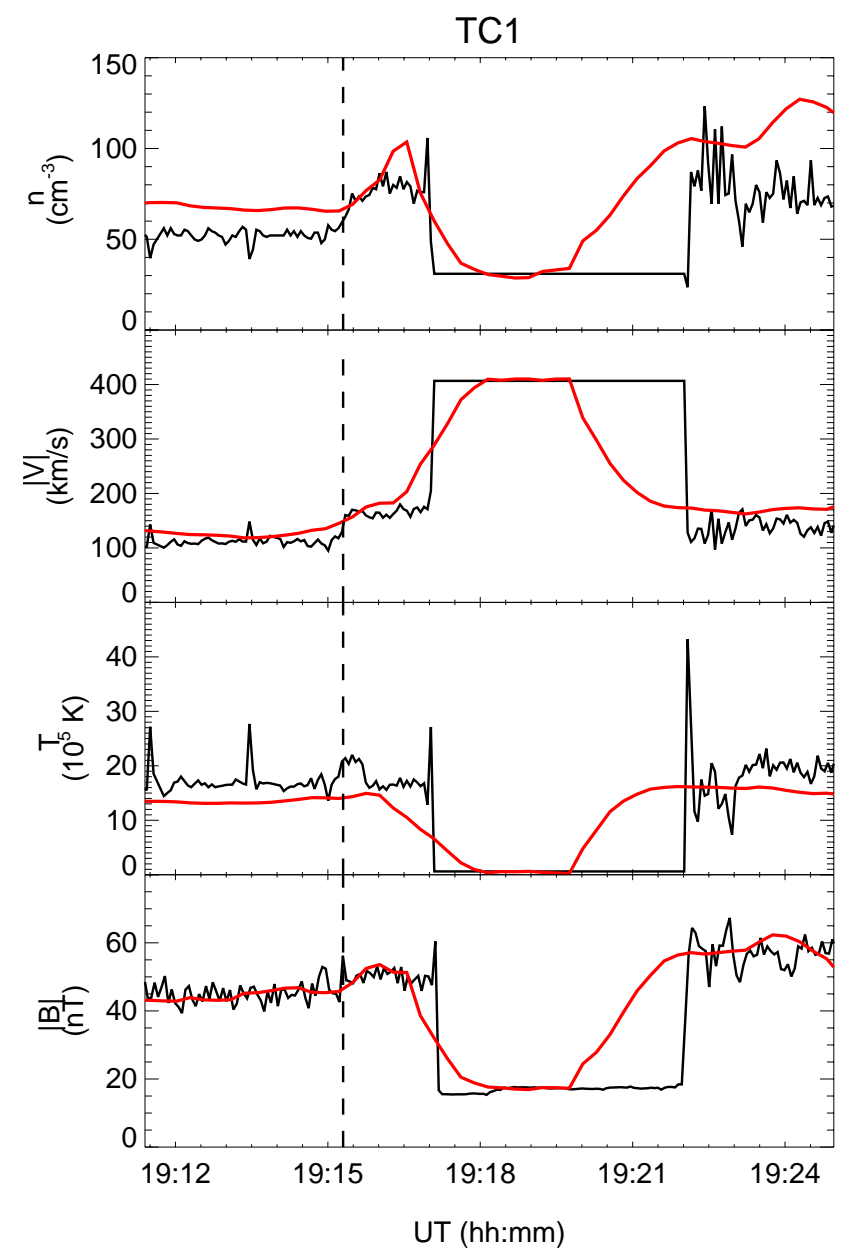

Fig. 7. Ions density, total velocity, temperature, and magnetic field magnitude observed by HIA and FGM instruments onboard TC1 (black lines) and predicted by the MHD code (red lines). Vertical dashed line marks the arrival of IS.

results were obtained by Samsonov et al. (2007) with a global MHD code. TC1 being at that time in the solar wind can not observe the RFS, but SC3 can observe the RFS as an increase of simulated $n, T, B$ and a decrease of simulated $V\left(\left|V_{\mathrm{x}}\right|\right.$ decreases $)$ starting at around 19:18:00 UT in Fig. 6. The reflected shock or wave results in the outward bow shock motion which creates another discontinuity moving inward through the magnetosheath. In Figs. 8-11, this discontinuity near the Sun-Earth line is characterized by a decrease of $n$ and $B$, and an increase of $T$ (panels 15-18). However, only a smooth decrease of simulated $n$ is obtained at the SC3 position beginning from about 19:19:30 UT in Fig. 6. Since SC3 is in the inner magnetosheath rather far from the SunEarth line, the simulated variations at its position are a mixture of inward and outward waves. A strict identification of this structure in the simulation is hardly possible and, therefore, a more strict analysis should be done in future works using the Rankine-Hugoniot conditions.

\section{Discussion}

The analysis of the combined observations of TC1 and SC3 has given some quantitative results which is useful to discuss and to highlight.

The IS speeds, measured in the Earth's reference frame, are $U_{\mathrm{n}_{(\mathrm{IS})}}(\mathrm{ACE})=461 \mathrm{~km} / \mathrm{s}$ and $U_{\mathrm{n}_{(\mathrm{IS})}}(\mathrm{TC} 1)=328 \mathrm{~km} / \mathrm{s}$, $U_{\mathrm{n}_{(\mathrm{IS})}}(\mathrm{SC} 3)=314 \mathrm{~km} / \mathrm{s}$ in the interplanetary medium and in the magnetosheath, respectively. Therefore, the IS is slowed down by the interaction with the bow shock as already reported in previous papers (Villante et al., 2004; Koval et al., 2006a). The IS deceleration here obtained is more close to the estimates by Koval et al. (2006a) than to the results by Villante et al. (2004). In fact, in the present case the magnetosheath speeds of IS are 0.71 and 0.68 of the corresponding interplanetary speed whereas Koval et al. (2006a) and Villante et al. (2004) have reported a value ranging from 0.82 to 0.97 and from 0.25 to 0.33 , respectively. Besides the Rankine-Hugoniot istantaneous estimates $U_{\mathrm{n}_{(\mathrm{IS})}}$ (TC1) and $U_{\mathrm{n}_{(\mathrm{IS})}}(\mathrm{SC} 3)$, an average value of $<U_{\mathrm{n}_{(\mathrm{IS})}}>=375 \mathrm{~km} / \mathrm{s}$ of the IS magnetosheath speed has been obtained using the time delay between the IS passages at TC1 and SC3 positions (we note that, considering as shock speed $<U_{\mathrm{n}_{(\mathrm{IS})}}>$, the IS deceleration is 0.82 , that is a value falling in the above mentioned Koval's interval). Taken into account that $\left\langle U_{\mathrm{n}_{(\mathrm{IS})}}>\right.$, $U_{\mathrm{n}_{(\mathrm{IS})}}(\mathrm{TC} 1)$ and $U_{\mathrm{n}_{(\mathrm{IS})}}(\mathrm{SC} 3)$ are all quite close, we can conclude that the travel through the magnetosheath does not further decelerates the IS. Moreover, going from the interplanetary space to the magnetosheath, the shock compression ratio, $r=\varrho_{2} / \varrho_{1}$, has a decrease from $r=1.6$ to $r=1.4$ and $r=1.5$, the last two values being the measurements of $r$ made at TC1 and SC3 locations, respectively. Therefore, the observations indicate that the IS is scarcely weakened by the collision with the bow shock and also by the following propagation through the magnetosheath.

As shown in Sect. 2, just after the IS, both TC1 and SC3 observe in the magnetosheath a second discontinuity having an increase of $n$ and a decrease of $T$. Moreover, no track of this discontinuity is found in solar wind data. In this regard, therefore, our observations are in qualitative agreement with the observations reported by Šafránková et al. (2007). In fact, analysing some others events similar to the present one, those authors have found that the magnetosheath perturbations, due to the impact of an IS on the bow shock, have a two-step like structure when observed by a spacecraft positioned very close to the bow shock (such as TC1 in the present case). The first step being the transmitted IS and the second step being a discontinuity strictly resembling that observed in the present event. Differently from Šafránková et al. (2007), we have given an estimate of the speed of this discontinuity finding a value of $164 \mathrm{~km} / \mathrm{s}$ very close to the plasma bulk flow speed along the discontinuity normal, i.e. this structure does not propagate with respect to the plasma. This finding is in good agreement with a quantitative result, obtained by (Přech et al., 2008), concerning the speed 
$\Delta \mathrm{n}\left(\mathrm{cm}^{-3}\right)$ in every $16 \mathrm{~s}$ from $\mathrm{t}=19: 15 \mathrm{UT}$
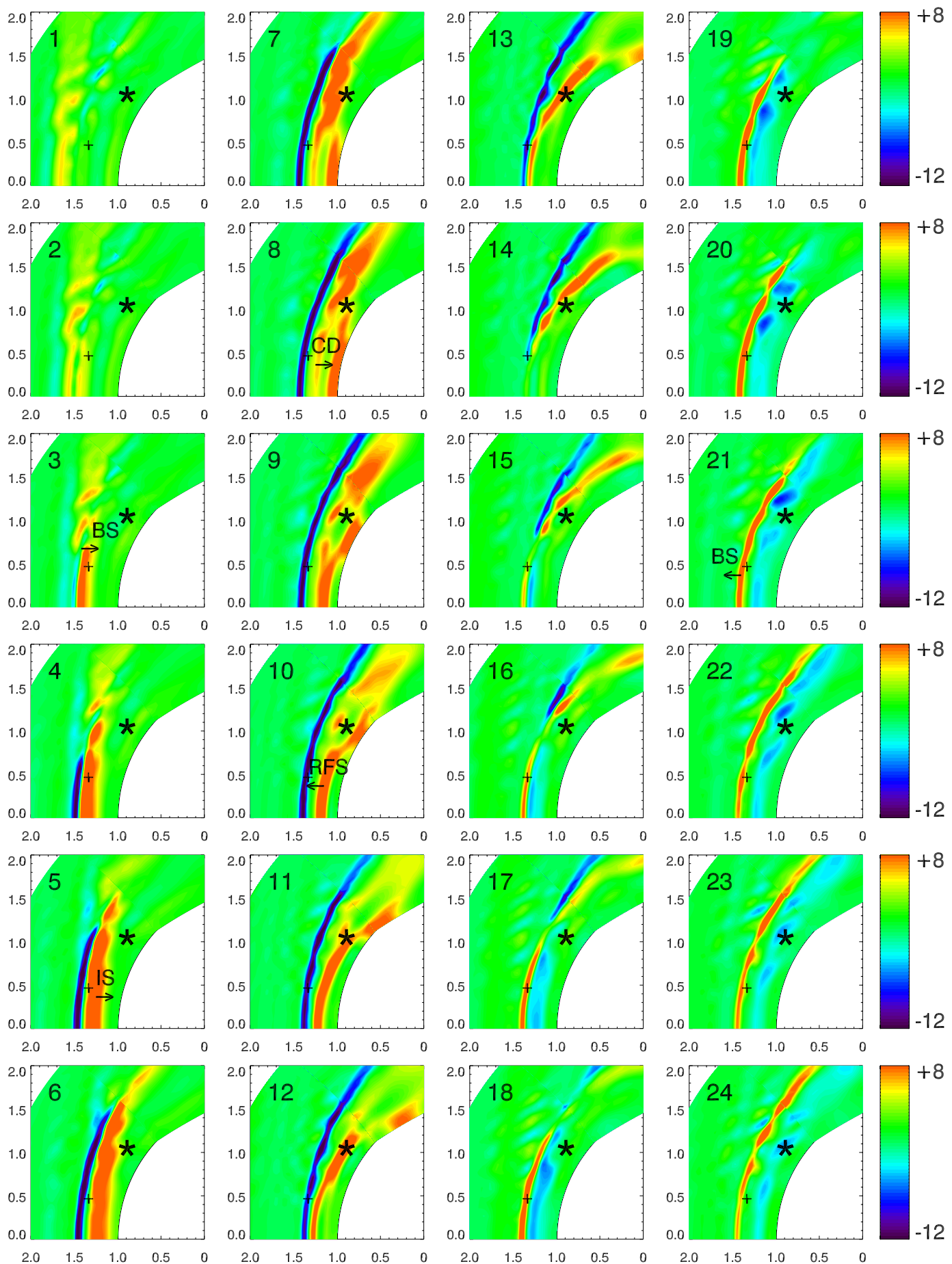

Fig. 8. Contours of $\Delta n$ in the noon-meridional plane for a successive set of 24 time intervals, where $\Delta n$ is a variation of density during $16 \mathrm{~s}$ interval. The abbreviations BS, IS, CD, and RFS refer to the bow shock, interplanetary shock, compound discontinuity, and reflected fast shock, respectively. A star and a cross in every panel illustrate relative positions of simulated SC3 and TC1 with respect to the magnetopause and bow shock. The Sun-Earth line is along the horizontal axis. 


\section{$\Delta V$ in every $16 s$ from $t=19: 15$ UT -7 May 2005}
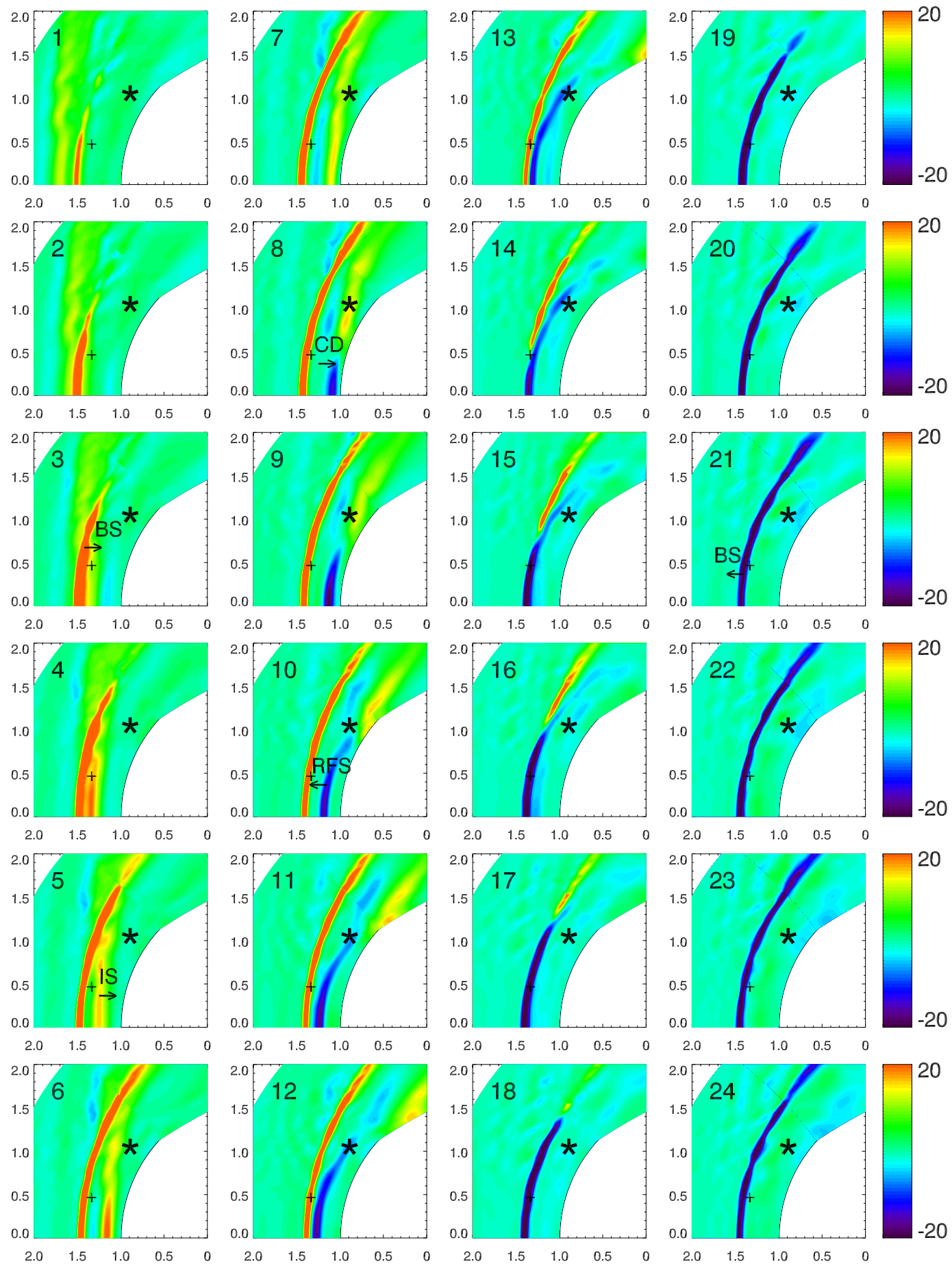

Fig. 9. Contours of $\Delta V$ showing variations of velocity. The format is the same as that in Fig. 8 .

of the discontinuity which follows the IS. It is very interesting to stress that the numerical simulations by Samsonov et al. (2006) are generally consistent with the observations reported in the present and Šafránková et al. (2007) and Přech et al. (2008) studies. Indeed, Samsonov et al. (2006) have found a compound discontinuity (comprising a forward slow expansion wave, a contact discontinuity and a reversed slow shock) which travels, at nearly the local plasma flow speed, 
$\Delta B$ in every $16 \mathrm{~s}$ from $t=19: 15$ UT - 7 May 2005
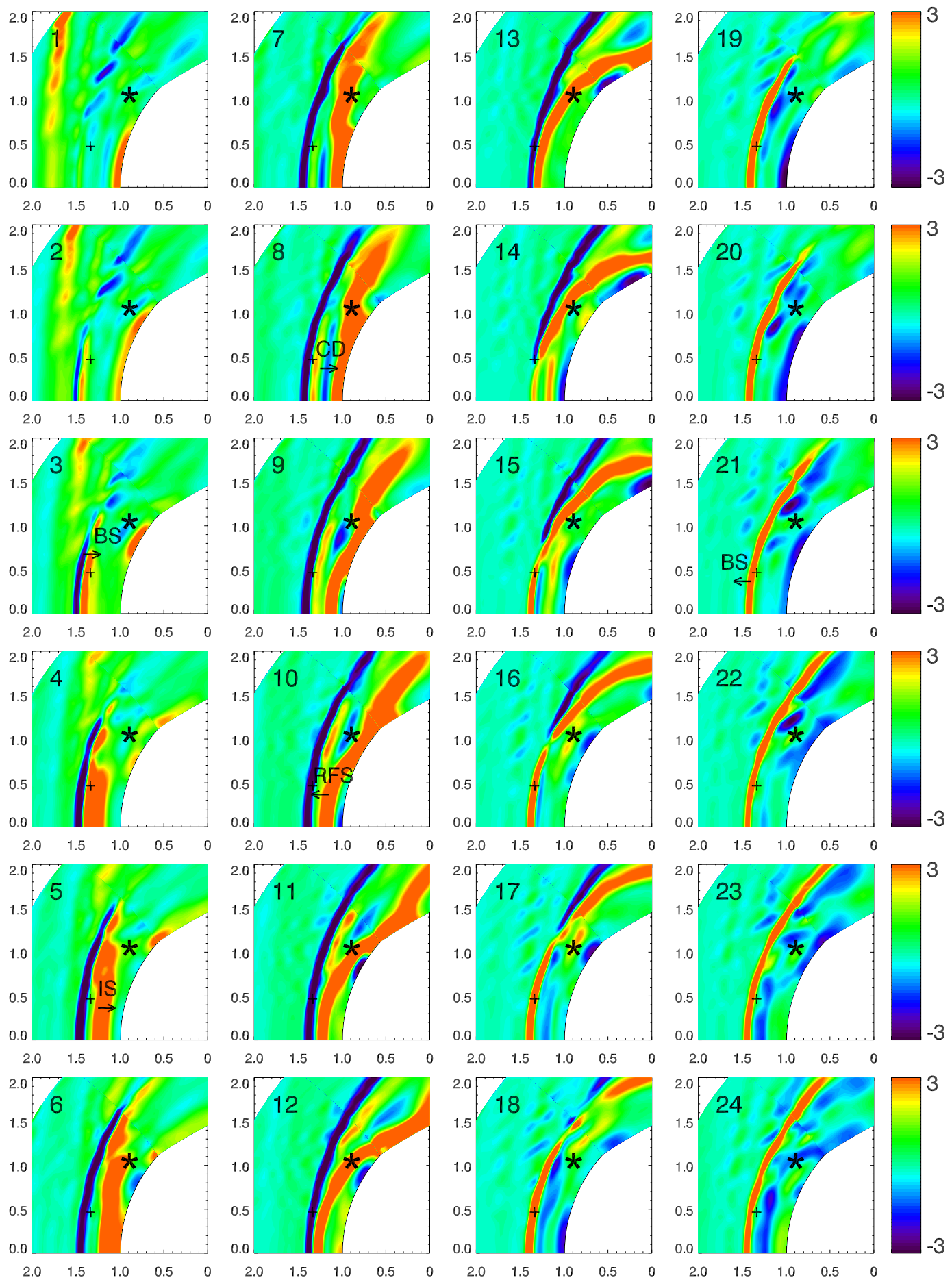

Fig. 10. Contours of $\Delta B$ showing variations of field magnitude. The format is the same as that in Fig. 8 .

behind the transmitted IS and which is generated by the interaction between the IS and the bow shock. This discontinuity is mainly charatherized by an increase of $n$, a decrease of $T$ and a weak increase of $B$.
A third discontinuity, actually a not propagating structure which moves earthward at the local plasma flow speed, is present in the magnetosheath perturbation seen by SC3. On the contrary, TC1 does not observe this discontinuity neither 
$\Delta T(K)$ in every $16 \mathrm{~s}$ from $\mathrm{t}=19: 15 \mathrm{UT}$
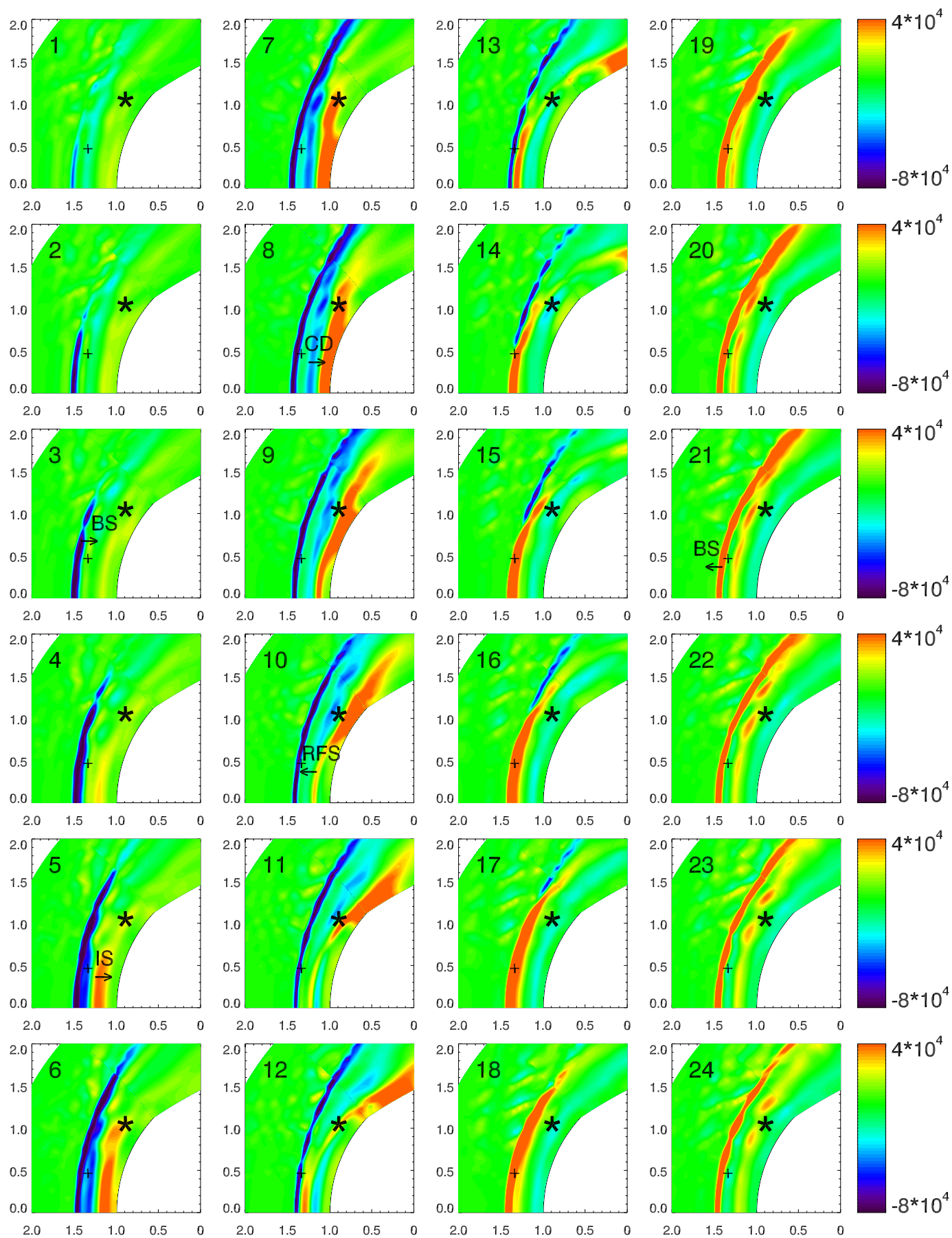

Fig. 11. Contours of $\Delta T$ showing variations of temperature. The format is the same as that in Fig. 8 .

in the magnetosheath nor in the solar wind. An inspection of the solar wind data excludes the interplanetary origin of this discontinuity and, therefore, it must be considered as a product of some process linked to the global IS-magnetosphere interaction. Note that, to our knowledge, this is the first time that such a kind of discontinuity is reported in observational studies regarding the present topic. With regard to the generation of this discontinuity, a possible qualitative explanation comes from the event numerical simulation. The latter, in fact, shows that the reflection of the transmitted IS 
from the magnetopause results in a fast reverse shock (RFS) moving sunward. When RFS hits the bow shock, this starts to move outward and, at the same time, a new discontinuity (earthward propagating) is produced. In the simulation, this discontinuity is characterized by a decrease of $n$ and $B$, and an increase of $T$ whereas the observed discontinuity shows an increase of $B$ (besides a decrease of $n$ and an increase of $T$ ), that is the simulation does not exactly fit the observations in this specific case. Anyway, in our opinion, the numerical simulation is very useful because it suggests a generation mechanism which is able to explain the reason why TC1 misses to observe this discontinuity. The hypothetical scenario could be the following: $\mathrm{TC} 1$ is in the solar wind when the RFS reaches the bow shock, later, the outward motion of the bow shock causes the inbound crossing of TC1 but, by this time, the discontinuity is far from the TC1 position due to its earthward motion through the magnetosheath. At this point, we must clarify an important question regarding the existence of the RFS. In the present simulation, as already reported at the beginning of Sect. 3, the magnetopause is modelled as a solid and impenetrable obstacle. As a matter of fact, such approximation does not permit to take properly into account the magnetopause motion due to the interaction with the IS. In fact, Grib et al. (1979) and $\mathrm{Wu}$ et al. (1993) have found that the IS impact upon the magnetopause produces a rarefaction wave rather than a RFS. However, we note that our local model gives, into the magnetosheath, results close to those obtained by Samsonov et al. (2007) (e.g. see their Figs. 5 and 6) by means of a global MHD code where the IS is reflected by a boundary placed inside the magnetosphere. As Samsonov et al. (2007) noted, this boundary is either the plasmapause or the ionosphere. Therefore, we are quite confident that a RFS is really produced, but it originates inside the magnetosphere and propagates outward through the magnetopause and magnetosheath.

Around 2 min after the passage of the transmitted IS at its position, TC1 has an outbound crossing of the bow shock followed, 5 min later, by an inbound crossing which brings back the spacecraft from the solar wind to the magnetosheath. The TC1 observations are, therefore, very similar to those discussed by Šafránková et al. (2007) with respect to some others events where spacecraft, located in very outer magnetosheath, observed the effects of an IS impact on the bow shock. Šafránková et al. (2007) have suggested, supported by 3-D MHD simulations based on Samsonov's model (Samsonov et al., 2006), that the first (outbound) crossing is due, as predicted by the theory (e.g., Grib et al., 1979), to an earthward displacement of the bow shock caused by its interaction with the IS, whereas the second (inbound) crossing is given by a RFS from the inner magnetosphere which pushes the bow shock outward when colliding with it. The above suggestions are completely confirmed by the special set simulation of our event. In fact, as described in Sect. 3, our simulation predicts correctly the TC1 double crossing and, moreover, shows as the inward and outward displacements of the bow shock are caused by the collisions on it of the IS and the reverse shock respectively. However, with regard to the $\mathrm{TC} 1$ double crossing the most interesting results come from the experimental data. In fact, we have been able to measure the bow shock speeds for both TC1 crossings $\left(U_{\mathrm{n}_{\left(\mathrm{BS}_{1}\right)}}=47 \mathrm{~km} / \mathrm{s}\right.$ and $\left.U_{\mathrm{n}_{\left(\mathrm{BS}_{2}\right)}}=80 \mathrm{~km} / \mathrm{s}\right)$ obtaining a clear observational confirmation that the bow shock has a definite motion, first earthward and, then, sunward directed. It is noted that the inward bow shock speed $U_{\mathrm{n}_{\left(\mathrm{BS}_{1}\right)}}=47 \mathrm{~km} / \mathrm{s}$ is close to the value of $\sim 40 \mathrm{~km} / \mathrm{s}$ obtained by Přech et al. (2008) studying a different event. Bow shock speeds as $U_{\mathrm{n}_{\left(\mathrm{BS}_{1}\right)}}$ and $U_{\mathrm{n}_{\left(\mathrm{BS}_{2}\right)}}$ are not so frequent. As a matter of fact, Šafránková et al. (2003) have reported that in a large majority of cases, i.e. $78 \%$ of the 112 bow shock crossings analyzed, the shock velocity is below $40 \mathrm{~km} / \mathrm{s}$. In the present case, moreover, there are no significant variations, in the solar wind plasma and the magnetic field following the IS front, such to justify the above crossings. Therefore, there exists a strong indication that the double bow shock displacement is somehow due to the interaction IS-magnetosphere. As shown in Sect. 2, under the hypothesis that the bow shock starts to move, from $\boldsymbol{R}_{\text {impact }}$, immediately after the interaction with the IS at the time $T_{\text {impact }}$, we have found that its speed should be $\sim 55 \mathrm{~km} / \mathrm{s}$, a value only $\sim 1.2$ higher than the direct measure of $U_{\mathrm{n}_{\left(\mathrm{BS}_{1}\right)}}=47 \mathrm{~km} / \mathrm{s}$. Therefore, taken into account the crudeness of some approximations made in this calculation, we can be quite confident that the observed earthward motion of the bow shock is really due to the interaction with IS and that $\boldsymbol{R}_{\text {impact }}$ and $T_{\text {impact }}$ are reliable estimates. Moreover, the reliability of $\boldsymbol{R}_{\text {impact }}$ and $T_{\text {impact }}$ is an indirect confirmation of the hypothesis on which the calculation of those reconstructed values is based on (Sect. 2), i.e. that the second discontinuity is generated by the collision of the IS upon the bow shock. Viceversa, our data do not permit to shed much more light on the origin of the sunward bow shock motion. As reported earlier, the MHD simulation of the event shows that the outward displacement of the bow shock is given by the interaction with a reverse shock or wave. Unfortunately, in the data we have found only an indication of the existence of this reverse wave but not any robust evidence. The indication is given by the smooth increases of $n$ and $P_{\text {th }}$ and by the regular decrease of $\left|V_{\mathrm{x}}\right|$ observed in $\mathrm{SC} 3$ data between 19:17:00 and 19:20:00 UT, that is just after the passage of the IS. These trends, in fact, are compatible with the transit of a compressive wave propagating sunward.

\section{Summary}

We have analyzed, by means of Double Star TC1 and Cluster SC3 data, an event of magnetosheath perturbation caused by the impact of IP shock on the terrestrial bow shock. We have also performed a 3-D numerical MHD simulation of this 
event to get some suggestions for its physical interpretation. Our results are summarized as follows:

1. The transmitted IS has a speed lower than the incident IS (in the Earth's reference frame) and is not further slowed down by the propagation into the magnetosheath. Moreover, the impact with the bow shock produces a negligible weakening of the IS strength.

2. Besides the transmitted shock, the interaction IS-bow shock produces also a second discontinuity which is moving earthward with the local bulk flow speed.

3. A third discontinuity, moving earthward with the local plasma flow speed, is also present but only in SC3 data. This discontinuity is not from the interplanetary space but is the product of some process linked to the global interaction IS-magnetosphere. The 3-D numerical simulation of the event suggests as a possible mechanism of its generation the interaction between a reverse fast shock and the bow shock.

4. After the passage of the IS, the bow shock moves first earthward and then sunward. Moreover, our observations provide a robust and experimental support to the theoretical prediction that the inward motion of the bow shock is due to its interaction with the incident IS. As the numerical simulation indicates, the outward displacement of the bow shock is produced, together with the third discontinuity, by its impact with a reverse shock coming from the inner magnetosphere. An observational indication (indeed quite vague) of the existence of the above reverse fast shock has been found in SC3 data.

Acknowledgements. The authors would like to thank the CLUSTER/FGM (PI: E. Lucek), the ACE/MAG (PI: N. F. Ness) and ACE/SWEPAM (PI: D. J. McComas) teams for making verified data available, the CDAWeb and CAA teams for providing online public access to ACE and CLUSTER datasets respectively. The work done at IFSI has been supported by Agenzia Spaziale Italiana (ASI) under contract ASI-INAF I/023/09/0 "Attività Scientifica per l'Analisi Dati Sole e Plasma - Fase E2".

Topical Editor I. A. Daglis thanks two anonymous referees for their help in evaluating this paper.

\section{References}

Akhiezer, A. I., Akhiezer, I. A., Polovin, R. V., Sitenko, A. G., and Stepanov, K. N.: Plasma electrodynamics - Vol.1: Linear theory; Vol.2: Non-linear theory and fluctuations, International Series of Monographs in Natural Philosophy, Oxford: Pergamon Press, 1975, 1975.

Balogh, A., Carr, C. M., Acuña, M. H., Dunlop, M. W., Beek, T. J., Brown, P., Fornacon, K.-H., Georgescu, E., Glassmeier, K.H., Harris, J., Musmann, G., Oddy, T., and Schwingenschuh, K.: The Cluster Magnetic Field Investigation: overview of in-flight performance and initial results, Ann. Geophys., 19, 1207-1217,
2001,

http://www.ann-geophys.net/19/1207/2001/.

Carlson, C. W., Curtis, D. W., Paschmann, G., and Michel, W.: An instrument for rapidly measuring plasma distribution functions with high resolution, Adv. Space Res., 2, 67-70, doi:10.1016/ 0273-1177(82)90151-X, 1982.

Carr, C., Brown, P., Zhang, T. L., Gloag, J., Horbury, T., Lucek, E., Magnes, W., O’Brien, H., Oddy, T., Auster, U., Austin, P., Aydogar, O., Balogh, A., Baumjohann, W., Beek, T., Eichelberger, H., Fornacon, K.-H., Georgescu, E., Glassmeier, K.-H., Ludlam, M., Nakamura, R., and Richter, I.: The Double Star magnetic field investigation: instrument design, performance and highlights of the first year's observations, Ann. Geophys., 23, 27132732, 2005, http://www.ann-geophys.net/23/2713/2005/.

Dryer, M.: Bow shock and its interaction with interplanetary shocks., Radio Science, 8, 893-901, 1973.

Formisano, V.: Orientation and shape of the earth's bow shock in three dimensions, Planet. Space Sci., 27, 1151-1161, doi: 10.1016/0032-0633(79)90135-1, 1979.

Grib, S. A.: Interaction of non-perpendicular/parallel solar wind shock waves with the earth's magnetosphere, Space Sci. Rev., 32, 43-48, 1982.

Grib, S. A. and Martynov, M. V.: Formation of a shock wave in the magnetosheath of the earth's magnetosphere, Geomagn. Aeron., 17, 252-258, 1977.

Grib, S. A. and Pushkar, E. A.: Asymmetry of nonlinear interactions of solar MHD discontinuities with the bow shock, Geomagn. Aeron., 46, 417-423, doi:10.1134/S0016793206040025, 2006.

Grib, S. A., Briunelli, B. E., Dryer, M., and Shen, W.-W.: Interaction of interplanetary shock waves with the bow shockmagnetopause system, J. Geophys. Res., 84, 5907-5921, 1979.

Hudson, P. D.: Discontinuities in an anisotropic plasma and their identification in the solar wind, Planet. Space Sci., 18, 16111622, doi:10.1016/0032-0633(70)90036-X, 1970.

Huttunen, K. E. J., Slavin, J., Collier, M., Koskinen, H. E. J., Szabo, A., Tanskanen, E., Balogh, A., Lucek, E., and Rème, H.: Cluster observations of sudden impulses in the magnetotail caused by interplanetary shocks and pressure increases, Ann. Geophys., 23, 609-624, 2005, http://www.ann-geophys.net/23/609/2005/.

Jeffrey, A. and Taniuti, T.: Non-linear wave propagation, Mathematics in Science and Engineering, New York: Academic Press, 1964, 1964.

Koval, A., Šafránková, J., Němeček, Z., Přech, L., Samsonov, A. A., and Richardson, J. D.: Deformation of interplanetary shock fronts in the magnetosheath, Geophys. Res. Lett., 32, L15101, doi:10.1029/2005GL023009, 2005.

Koval, A., Šafránková, J., Němeček, Z., and Přech, L.: Propagation of interplanetary shocks through the solar wind and magnetosheath, Adv. Space Res., 38, 552-558, doi:10.1016/j.asr.2006. 05.023, 2006a.

Koval, A., Šafránková, J., Němeček, Z., Samsonov, A. A., Přech, L., Richardson, J. D., and Hayosh, M.: Interplanetary shock in the magnetosheath: Comparison of experimental data with MHD modeling, Geophys. Res. Lett., 33, L11102, doi:10.1029/ 2006GL025707, 2006b.

Landau, L. D. and Lifshitz, E. M.: Electrodynamics of continuous 
media, Pergamon Press, 1960.

Pushkar', E. A., Barmin, A. A., and Grib, S. A.: Investigation in the MHD approximation of the incidence of the solar-wind shock wave on the near-earth bow shock, Geomagn. Aeron., 31, 522$525,1991$.

Přech, L., Němeček, Z., and Šafránková, J.: Response of magnetospheric boundaries to the interplanetary shock: Themis contribution, Geophys. Res. Lett., 35, 17, doi:10.1029/2008GL033593, 2008.

Rème, H., Aoustin, C., Bosqued, J. M., Dandouras, I., Lavraud, B., Sauvaud, J. A., Barthe, A., Bouyssou, J., Camus, Th., CoeurJoly, O., Cros, A., Cuvilo, J., Ducay, F., Garbarowitz, Y., Medale, J. L., Penou, E., Perrier, H., Romefort, D., Rouzaud, J., Vallat, C., Alcaydé, D., Jacquey, C., Mazelle, C., d'Uston, C., Möbius, E., Kistler, L. M., Crocker, K., Granoff, M., Mouikis, C., Popecki, M., Vosbury, M., Klecker, B., Hovestadt, D., Kucharek, H., Kuenneth, E., Paschmann, G., Scholer, M., Sckopke, N., Seidenschwang, E., Carlson, C. W., Curtis, D. W., Ingraham, C., Lin, R. P., McFadden, J. P., Parks, G. K., Phan, T., Formisano, V., Amata, E., Bavassano-Cattaneo, M. B., Baldetti, P., Bruno, R., Chionchio, G., Di Lellis, A., Marcucci, M. F., Pallocchia, G., Korth, A., Daly, P. W., Graeve, B., Rosenbauer, H., Vasyliunas, V., McCarthy, M., Wilber, M., Eliasson, L., Lundin, R., Olsen, S., Shelley, E. G., Fuselier, S., Ghielmetti, A. G., Lennartsson, W., Escoubet, C. P., Balsiger, H., Friedel, R., Cao, J.-B., Kovrazhkin, R. A., Papamastorakis, I., Pellat, R., Scudder, J., and Sonnerup, B.: First multispacecraft ion measurements in and near the Earths magnetosphere with the identical Cluster ion spectrometry (CIS) experiment, Ann. Geophys., 19, 1303-1354, 2001,

http://www.ann-geophys.net/19/1303/2001/.

Rème, H., Dandouras, I., Aoustin, C., Bosqued, J. M., Sauvaud, J. A., Vallat, C., Escoubet, P., Cao, J. B., Shi, J., BavassanoCattaneo, M. B., Parks, G. K., Carlson, C. W., Pu, Z., Klecker, B., Moebius, E., Kistler, L., Korth, A., Lundin, R., and the HIA team: The HIA instrument on board the Tan Ce 1 Double Star near-equatorial spacecraft and its first results, Ann. Geophys., 23, 2757-2774, 2005, http://www.ann-geophys.net/23/2757/2005/.

Šafránková, J., Jelínek, K., and Němeček, Z.: The bow shock velocity from two-point measurements in frame of the interball project, Adv. Space Res., 31, 1377-1382, 2003.

Šafránková, J., Němeček, Z., Přech, L., Samsonov, A. A., Koval, A., and Andréeová, K.: Modification of interplanetary shocks near the bow shock and through the magnetosheath, J. Geophys. Res., 112, A08212, doi:10.1029/2007JA012503, 2007.
Samsonov, A.: Numerical modelling of the Earth magnetosheath for different IMF orientations, Adv. Space Res., 38, 1652-1656, doi:10.1016/j.asr.2005.06.009, 2006.

Samsonov, A. A., Němeček, Z., and Šafránková, J.: Numerical MHD modeling of propagation of interplanetary shock through the magnetosheath, J. Geophys. Res., 111, A08210, doi:10.1029/ 2005JA011537, 2006.

Samsonov, A. A., Sibeck, D. G., and Imber, J.: MHD simulation for the interaction of an interplanetary shock with the Earth's magnetosphere, J. Geophys. Res., 112, A12220, doi: 10.1029/2007JA012627, 2007.

Schwartz, S. J.: Shock and Discontinuity Normals, Mach Numbers, and Related Parameters, Analysis Methods for Multi-Spacecraft Data, edited by: Paschmann, G. and Daly, P., ISSI Scientific Reports Series, ESA/ISSI, 1, 249-270, 1998.

Shen, W.-W. and Dryer, M.: Magnetohydrodynamic theory for the interaction of an interplanetary double-shock ensemble with the earth's bow shock., J. Geophys. Res., 77, 4627-4644, 1972.

Sonnerup, B. U. O. and Cahill Jr., L. J.: Magnetopause Structure and Attitude from Explorer 12 Observations, J. Geophys. Res., 72, 171, doi:10.1029/JZ072i001p00171, 1967.

Spreiter, J. R. and Stahara, S. S.: Gasdynamic and magnetohydrodynamic modeling of the magnetosheath: A tutorial, Adv. Space Res., 14, 5-19, doi:10.1016/0273-1177(94)90042-6, 1994.

Tóth, G.: Comparison of Some Flux Corrected Transport and Total Variation Diminishing Numerical Schemes for Hydrodynamic and Magnetohydrodynamic Problems, J. Comp. Phys., 128, 82100, doi:10.1006/jcph.1996.0197, 1996.

Tsurutani, B. T. and Gonzalez, W. D.: The Interplanetary Causes of Magnetic Storms: A Review, pp. 77-90, Magnetic Storms, Geophysical Monograph Series, Vol. 98, 1997.

Villante, U., Lepidi, S., Francia, P., and Bruno, T.: Some aspects of the interaction of interplanetary shocks with the Earth's magnetosphere: an estimate of the propagation time through the magnetosheath, J. Atmos. Terr. Phys., 66, 337-341, doi: 10.1016/j.jastp.2004.01.003, 2004.

Wu, B., Mandt, M. E., Lee, L. C., and Chao, J. K.: Magnetospheric response to solar wind dynamic pressure variations: Interaction of interplanetary tangential discontinuities with the bow shock, J. Geophys. Res., 98, 21297, doi:10.1029/93JA01013, 1993.

Yan, M. and Lee, L. C.: Interaction of interplanetary shocks and rotational discontinuities with the Earth's bow shock, J. Geophys. Res., 101, 4835-4848, doi:10.1029/95JA02976, 1996. 